\title{
Polysèmes
}

Revue d'études intertextuelles et intermédiales

\section{"Vampirella » et " The Lady of the House of Love » : transformations métamorphiques, transpositions métaphoriques}

\section{Stéphanie Amar-Flood}

\section{(2) OpenEdition}

Journals

\section{Édition électronique}

URL : http://journals.openedition.org/polysemes/1626

DOI : 10.4000/polysemes. 1626

ISSN : 2496-4212

Éditeur

SAIT

Édition imprimée

Date de publication : 1 janvier 2003

Pagination : 149-161

ISSN : 0999-4203

\section{Référence électronique}

Stéphanie Amar-Flood, « «Vampirella » et « The Lady of the House of Love » : transformations métamorphiques, transpositions métaphoriques », Polysèmes [En ligne], 6 | 2003, mis en ligne le 10 janvier 2017, consulté le 10 décembre 2020. URL : http://journals.openedition.org/polysemes/1626 ; DOI : https://doi.org/10.4000/polysemes.1626

Ce document a été généré automatiquement le 10 décembre 2020.

Polysèmes 


\title{
"Vampirella » et « The Lady of the House of Love » : transformations métamorphiques, transpositions métaphoriques
}

\author{
Stéphanie Amar-Flood
}

Dans sa célèbre étude sur l'aphasie, où il postule que le langage s'organise autour de deux pôles - l'un qu'il qualifie de métaphorique, auquel il associe la similarité, la sélection et la substitution, et l'autre marqué par les figures de la contiguïé, la combinaison et la contexture, qu'il nomme métonymique -, Jakobson suggère que ce principe duel est à la base de bien plus que le fonctionnement du langage. Selon son hypothèse, toute production culturelle oscille entre ces deux modes, bien que chacune entretienne plus ou moins d'affinité avec l'un ou l'autre. Ainsi dans le domaine des arts plastiques, où l'on décèle des mouvements divergents dans des styles qui se sont pourtant liés d'un point de vue temporel, on discerne également une divergence de tendances: " on peut noter l'orientation manifestement métonymique du cubisme, qui transforme l'objet en une série de synecdoques ", écrit Jakobson, en ajoutant que «les peintres surréalistes ont réagi par une conception visiblement métaphorique $»^{1}$. Dans le domaine de la littérature, c'est la poésie, "gouvernée par un principe de similarité », qui se révèle essentiellement d'ordre métaphorique, tandis que la prose « se meut essentiellement dans les rapports de contiguité $»^{2}$, et manifeste de ce fait une affinité avec la métonymie. L'art dramatique, toujours selon Jakobson, entre en corrélation avec la poésie, donc avec le mode métaphorique ; le texte narratif, et surtout le texte réaliste, se fait remarquer par la forte prédominance $\mathrm{du}$ procès métonymique. Ce classement, faut-il le rappeler, a eu une influence considérable sur la critique littéraire pendant les années suivant sa publication, malgré (ou peut-être grâce à) son aspect « binariste » quelque peu réducteur 3 .

2 L'opposition que Jakobson établit entre le dramatique et le narratif constitue une distinction qui remonte aux conceptions classiques du rhétorique. Chez Aristote, par 
exemple, la diegesis et la représentation directe que donne l'art dramatique sont les deux volets de l'imitation poétique, la mimesis. Angela Carter rejoint pourtant Jakobson lorsqu'elle affirme que le récit, étant essentiellement de nature combinatoire, suit un axe métonymique : « [Any] narrative [...] will extend through time in, more or less, a straight line, its course determined by the characteristic copulas of the story: "and then[...]" "but then[...]" »; elle associe également la métaphore à l'art dramatique dans une courte remarque à propos de la première de ses cinq pièces radiophoniques : "Vampirella" is about vampirism as metaphor $»^{4}$. C'est précisément le rapport de cette pièce à une nouvelle qu'elle a engendrée, "The Lady of the House of Love" qui retient notre attention ici ${ }^{5}$. Bon nombre de remarques que Carter formule en guise d'explications au sujet de ses propres pièces radiophoniques laissent entendre que l'art dramatique en général, mais surtout la radio, constitue un genre plus propice à l'épanouissement de la métaphore que tout autre genre narratif, qu'il s'agisse de la nouvelle ou du roman. En prenant comme corpus "Vampirella » et "The Lady of the House of Love », cet article se donne pour objet de contester l'hypothèse selon laquelle le récit ne partage pas le même degré de figuralité que la pièce.

3 À première vue, la proximité temporelle de leur composition, ainsi que leur thématique commune, conduisent le critique à considérer la pièce et la nouvelle comme deux variantes d'une seule histoire présentée sous deux déclinaisons, le recto et le verso d'un ruban de Mœbius qui tourne sur lui-même. Sans remettre en cause le fait qu'il s'agit de deux actes scripturaires indépendants et distincts, l'on est néanmoins tenté de penser qu'ils sont motivés par une même impulsion créative, que pièce et nouvelle constituent une double refonte, dans des moules différents, de la même diégèse. Mais en dépit d'un indéniable chevauchement - certaines lignes de la pièce sont importées in extenso dans la nouvelle -, on discerne de nombreuses différences, et Carter elle-même insiste autant sur la spécificité de chacune d'entre elles que sur leurs points communs; sa remarque selon laquelle " each is quite different » contrarie le désir fédérateur de rapprocher les deux et d'y voir des liens. Carter insiste également sur la primauté de «Vampirella » en termes autres que ceux de la chronologie de composition (quatre ans séparent la publication de la nouvelle de la diffusion de la pièce). Si la pièce semble dotée d'une naissance parthénogénétique - " "Vampirella" came to me as radio, with all its images ready formed, in terms of words and sounds »-, Carter met l'accent sur le geste maïeutique qui a donné naissance à la nouvelle. La pièce est dès lors conceptualisée en "raw material » vampirisé par la plume romancière ; en ce sens, elle n'a pas influencé la nouvelle autant qu'elle lui a fourni les conditions de son écriture. C'est à partir de ce « cru» que le récit, plus soigné dans sa conception, plus « cuit » dans son produit, devient possible.

C'est d'ailleurs en termes d' « adaptation » et non de "reformulation » que l'auteur conçoit ce passage du mode dramatique au mode narratif. La distinction est d'importance, car si ce dernier présuppose la notion de synonymie, évoque la possibilité de dire la même chose mais de façon différente, il n'en va pas de même pour l'adaptation, qui contient, dans ce mouvement de transposition d'un genre littéraire vers un autre, un travail de mise en forme et d'arrangement, avec tout ce que cela entraîne en matière de réécriture. Une adaptation implique ainsi que la forme a une incidence non négligeable sur le contenu, que le logos est dépendent de la lexis : dire quelque chose autrement revient à dire autre chose. Il est clair que Carter fut curieuse d'explorer les différences artistiques entre le narratif et le dramatique et de cerner les modifications qu'une transition vers un texte narratif impose. Ce travail d'adaptation se conçoit d'ailleurs comme pur exercice de 
style: "to see what would and would not work in terms of prose fiction ». Le genre narratif, au moins dans sa guise de conte gothique, s'avère plus rigide que le mode dramatique et les changements sont exprimés à la fois en termes de restriction et de limitation. Plus sujet au poids des conventions littéraires, plus limitatif dans les possibilités qu'il ouvre à l'écrivain, le texte narratif s'oppose à l'« open-endedness » d'une pièce radiophonique et nécessite l'élagage de nombreux éléments qui font partie intégrante de la pièce maîtresse. C'est ainsi au risque d'une perte que la pièce devient récit. Le rétrécissement qui résulte de ce passage d'un mode à l'autre s'explique en partie par les règles afférant au genre de la nouvelle («the short story did not have sufficient space to discuss the nature, real and imagined of vampirism »), le souci de veiller à une certaine concision, des considérations concernant l'unité esthétique, la nécessité de maintenir une trame narrative; mais ces contraintes n'expliquent pas tous les changements estimés nécessaires.

5 Même si le récit s'appuie globalement sur les mêmes éléments diégétiques que ceux de la pièce - les tourments d'une femme vampire que seul le baiser d'un puceau peut libérer d'une existence de mort-vivant-, il introduit des différences d'envergure. La représentation de la comtesse, la Vampirella éponyme de la pièce, la comtesse Nosferatu dans la nouvelle, ainsi que celle du jeune soldat, n'est pas la même dans les deux cas. Misuccube, mi-enfant maudit, suspendue entre la vie et la mort, rongée par une éternelle insatisfaction, la jeune Vampirella est marquée d'une ambiguïté qui déstabilise l'auditeur, qui se demande, tout comme le jeune héros intrépide d'ailleurs, si elle est réellement un vampire, ou simplement la victime d'une curieuse psychopathologie familiale ${ }^{6}$. Le scepticisme de l'auditeur est doublé du pragmatisme inné et du bon sens du héros, qui toutefois n'est pas immunisé contre la peur, ressentant des frissons et souffrant d'une anxiété croissante : «I cannot in the least subdue a trembling in my hands ». En revanche, le narrateur du conte dépeint la comtesse Nosferatu comme un vampire vorace et sanguinaire, dont la vraie nature est incompréhensible pour le héros de par son manque d'expérience et son innocence. Si, dans sa naïveté, il l'estime «the hapless victim of inbreeding ", le lecteur la sait tout autre. Le héros de la nouvelle agit dans un monde à part, fait confiance à sa rationalité et à sa raison, et n'éprouve ni peur ni doute : «the young man [...] did not shiver in the blast of cold air »; "though he feels unease, he cannot feel terror $"$.

6 Autre différence de taille : la pièce est fermement ancrée dans un contexte historique, si bien que l'on peut même déterminer que les événements sont censés dater de la fin du mois de juin 1914 - dès son retour à Bucarest à la fin de la pièce, le héros est informé de l'assassinat de l'archiduc François-Ferdinand d'Autriche ; en revanche, le conte est dénué de tout élément qui permettrait de le fixer dans un contexte temporel précis, se déroulant « one hot ripe summer in the pubescent years of the present century ». La pièce intègre en outre des éléments de burlesque dans un thème qui semble au premier abord relever de l'épouvante: "I shall have to call on all my sang froid to deal with the situation " remarque le héros en prenant conscience des pulsions hématophages de la belle Vampirella. Composer pour les ondes semble faciliter un équilibre entre des genres a priori incompatibles; écrire d'un point de crête, sans tomber ni dans le mélodrame ni dans la comédie, par le maintien de « a knife-edge tension between black comedy and bizarre pathos ». Même les noms de certains personnages portent un élément d'humour : par homonymie, le nom Beane évoque ce qui n'est plus (fort propice dans le contexte d'une pièce sur les esprits et les vampires) ; bien que dans le cas du personnage de Blot, il 
s'agisse d'un caractère modelé sur un véritable sujet historique, son nom s'avère particulièrement riche en connotations, évoquant le sang ou tout liquide qui déborde et qui tache ${ }^{7}$. La nouvelle, pour sa part, est presqu'entièrement dénuée de tels éléments.

$7 \quad$ Les contraintes imposées par le passage au genre de la nouvelle sont donc en premier lieu d'ordre structurel, mais elles sont sans doute moins déterminantes que d'autres, qui rétrécissent ce que Carter nomme «l'espace imaginaire »: "Radio always leaves that magical and enigmatic margin, that space of the invisible, which must be filled in by the imagination of the reader ». C'est effectivement cette "marge », cet "espace», qui permettait de développer les possibilités métaphoriques dans la pièce, d'explorer les analogies et les résonances du vampirisme, de traiter le vampirisme en tant que métaphore, plutôt qu'en instance génératrice de métaphore. Si la pièce ne tente pas de répondre à la question " qu'est-ce que le vampirisme? », elle entreprend néanmoins un examen d'une variété de situations aux allures vampiriques. Le vampirisme dans la pièce n'est pas concept de base donnant lieu à des avatars, mais succédané, déjà figure métaphorisée. La prédation, le cannibalisme, la nécrophilie, le système capitaliste sont autant d'exemples de simulacres sans instance originale.

8 Écrire pour la radio implique d'ailleurs une narration à trois dimensions, qui contraste avec l'essentielle linéarité du récit. Son attrait réside précisément dans la possibilité de superposer plusieurs voix et de ponctuer, ou accompagner, ces discours d'effets sonores, de les coupler à des plages de silence. Le palimpseste d'éléments à la fois distincts et complémentaires, à l'image d'une partition musicale, confère à la pièce une qualité polyphonique qui est entièrement absente de la nouvelle. L'œuvre radiophonique prend effectivement forme par le biais d'un tourbillon de voix parfois littéraires (celle du comte Dracula), parfois historiques (le cannibale écossais Sawney Beane, le nécrophile français Henri Blot, la comtesse hongroise Elizabeth Ba'thory). Ce mélange discursif a pour premier effet de décentrer l'action géographiquement, en transportant l'auditeur tantôt dans un château dans les Carpates, tantôt vers le littoral écossais, tantôt dans une salle d'audience parisienne ; en faisant entendre une pluralité d'opinions sur des phénomènes de phagocytage, il introduit un véritable dialogisme. Si ces personnages témoignent d'une certaine extranéité vis-à-vis de la trame de l'histoire elle-même, ils sont tous liés à Vampirella soit par parenté littéraire, soit par leur goût pour l'ignoble ${ }^{8}$. Ces ancêtres de Vampirella, tels des âmes en perdition, envahissent la pièce ; leurs portraits suspendus aux murs du château pour surveiller le dernier fruit de leur lignée commentent et jugent, châtient et rassurent, confortant Vampirella dans sa dépravation. À l'image des textes chantés par les chœurs antiques, les paroles qu'énoncent ces damnés constituent un moyen (en l'occurrence dramatique) d'éviter le recours répété aux apartés ou soliloques des protagonistes, mais ils ne créent pas pour autant un effet de continuité et de cohésion. L'effet qui en résulte est plutôt celui d'un d'éclatement. Leurs interventions brisent la trame narrative de la pièce, et ralentissent la diégèse, provoquent une sorte d'effet brechtien de "distanciation» (Verfremdungseffekt). Mais paradoxalement, cette pluralité de voix focalise l'attention de l'auditeur sur le corps : sur la respiration et le ton, sur le débit, le rythme et l'élocution. Les paroles font entendre des accents prononcés - des inflexions écossaises pour le couple Beane - voire des langues étrangères : les premières paroles de Blot sont en français («chacun a son goût. Moi je préfère les cadavres »); une remarque de Vampirella fusionne anglais et allemand: "How, as the Germans say, gemütlich ». Ces voix sont les voix de l'innommable, de ceux qui sont exclus de la société civilisée et policée, et symbolisent le retour du réprimé dans cette confusion 
de voix babéliques et archaïques. Tous des exilés, tous des parias - tous sont bannis du texte de la nouvelle, même celui qui doit être le plus emblématique des vampires : «Even Vampirella's father, Count Dracula himself, was forced to bow out ».

9 La transposition en nouvelle entraîne un apaisement de cette dimension dialogique emblématique de la pièce. La polyphonie s'estompe, le chœur des voix qui tendait parfois vers une cacophonie disparaît. La fonction narrative qui circule si librement dans «Vampirella » est ainsi restreinte dans la nouvelle à un narrateur extradiégétique (et sporadiquement deux narrateurs intradiégétiques, la comtesse et le jeune soldat). La loquace Mrs Beane de la pièce renaît en gardienne muette, qui ne communique que par gestes. Le passage au récit implique en fait une décarnalisation autant qu'une décarnavalisation. Carter remarque de façon éclairante que la nouvelle est "leaner ", dégraissée du superflu, qu'elle tire sa cohérence esthétique de ses échos internes et non des résonances (ou autres échos) qu'elle déclenche.

10 Le corps n'est présent dans le conte que dans son absence : le miroir accroché au mur de la chambre du vampire ne renvoie que du vide ("does not reflect a presence»); des mânes (« intangibles ») hantent le jardin du château ; les revenants du village « manifest their presence by shadows ", la comtesse elle-même est « a cave full of echoes "; « a girl with the fragility of the skeleton of a moth, so thin, so frail that her dress seemed to him to hang suspended, as if untenanted in the dark air, a fabulous lending, a self-articulating garment in which she lived like a ghost in a machine. »

11 Dans un mouvement parallèle, la transposition de cette histoire dans le genre gothique implique une retextualisation, qui la noue à un intertexte littéraire plus riche et plus dense que celui de la pièce. À mesure que les références littéraires s'épaississent, le corps au contraire s'efface ; le corps vivace et volontaire de la pièce est condamné au silence d'un jeu de répétitions écholaliques. La pluralité de voix radiophoniques, qui contribue tant à l'épaisseur et à la vitalité de la pièce, s'amenuise à un simple écho; et même cette voix est retirée au protagoniste pour être attribuée au narrateur anonyme :

Her voice is filled with distant sonorities, like reverberations in a cave : now you are at the place of annihilation, now you are at the place of annihilation. [...] she is a system of repetitions, she is closed circuit [...] Her voice with the rushing sonorities of the ocean in it, a voice that seems to come elsewhere and from her white, still throat [...] Her voice is curiously disembodied. ${ }^{9}$

12 La vie que Vampirella est censée retirer de ses victimes en leur suçant le sang se voit ainsi retirée d'elle-même par un narrateur anonyme; la femme-vampire devient poupée mécanique, l'ensemble vide si archétypique de la féminité dans le canon littéraire. C'est donc une textualité qui prend la place de la texturalité propre à la radio, une textualité qui va de pair avec une décarnalisation. Il est particulièrement significatif que les personnages historiques de la pièce (Blot et Beane) disparaissent. Mrs Beane est dépouillée de son nom, et devient "her keeper ", ou «the crone». Le conte substitue l'anonymat à la diversité très physique des personnages de la pièce. Bon nombre de références littéraires sont explicites. Les contes de fées constituent une source intertextuelle riche, et le récit peut se lire comme une inversion de La Belle au bois dormant : le baiser du héros donne la mort ${ }^{10}$. Quelques lignes - en l'occurrence, le chant du géant sont empruntées à Jack et le haricot géant: "Fee fie fo fum/I smell the blood of an Englishman/Be he alive or be he dead/I'll grind his bones to make my bread ». Alice au pays des merveilles apparaît dans une brève réflexion du narrateur - «curiouser and curiouser ». Dès l'incipit, le lecteur ressent la présence de The Wasteland, (surtout 
The Burial of the Dead) dans une bribe de phrase (" too many shadows, even at midday "), une présence qui se confirme tout au long du récit par des éléments variés, la lecture du Tarot de la comtesse évoquant celle de Madame Sosostris n'étant que la plus évidente. Comme toujours, il y a un clin d'œil à Shakespeare, ici sous la forme de la description de la chambre de la comtesse, assimilée à "Juliet's tomb». D'autres détails ont l'effet de déclencher une chaîne d'associations chez le lecteur, telle la robe de mariée («antique bridal gown ») que porte la comtesse dans "The Lady of the House of Love ». L'état de délabrement du château, ainsi que les rideaux fermés en permanence renvoient clairement à l'apparence d'un autre habitant d'un manoir où l'amour ne réside plus, Miss Havisham de Great Expectations ${ }^{11}$. Les références aux automates - «her voice is curiously disembodied; she is like a doll, he thought, a ventriloquist's doll, or, more, like a great, ingenious piece of clockwork »; «the mechanism within her fails her, now, when she needs it most»- rappellent Olympia dans Der Sandmann, de E.T.A. Hoffmann. L'intertexte est pourtant plus large que ces simples renvois littéraires: ainsi le pictural est-il présent dans les références aux tableaux médiévaux de La Jeune Fille et la mort et l'opéra dans la description de la comtesse comme « queen of the night ».

La nouvelle qui sort pour ainsi dire des entrailles de la pièce, dépouillée de toute dimension dialogique, pourrait sembler quelque peu appauvrie et affaiblie. Or, il n'est plus question ici de traiter le vampirisme en tant que métaphore, de parcourir des chaînes de résonances ni de sonder des manifestations différentes de relations vampiriques. Le récit considère le vampirisme comme principe d'organisation dans le contexte plus large $d u$ genre du gothique ${ }^{12}$. En tant que tel, il dépend non seulement d'" effets de gothique", mais également de la description, qui, comme le rappelle G. Genette, constitue un élément incontournable pour tout récit ${ }^{13}$. C'est au sein des passages descriptifs que l'on voit surgir son intense métaphoricité. Le récit a nécessairement recours à la métaphore sans pour autant la prendre pour objet. De par le fait qu'il est pris dans les entrelacs d'un riche intertexte, le récit ne peut contourner la métaphore - celle de la rose est d'ailleurs longuement développée dans « The Lady of the House of Love ». Les deux axes orthogonaux que propose Jakobson prennent des allures de Scylla et Charybde et semblent nous conduire dans des eaux fort turbulentes. Il parait plus pertinent d'affirmer qu'il y a du métonymique dans la métaphore, tout comme du métaphorique dans la métonymie ${ }^{14}$. En dernière analyse, il apparait donc impossible de soustraire le conte du domaine de la métaphore ${ }^{15}$. Mais à un niveau encore plus fondamental, tel que la recherche post-structuraliste (post-Jakobson) l'illustre, il est impossible d'évacuer du discours la métaphore, de parler de la métaphore autrement que métaphoriquement. Comme le signale Ricœur, « Il n’y a pas de discours sur la métaphore qui ne se dise dans un réseau conceptuel lui-même engendré métaphoriquement. Il n'y a pas de lieu non métaphorique d'où l'on aperçoive l'ordre et la clôture du champ métaphorique. La métaphore se dit métaphoriquement ${ }^{16} \%$. Le passage d'un genre littéraire à un autre ne peut nullement occulter cet aspect métaphorique. 


\section{BIBLIOGRAPHIE}

Carter, Angela. "Vampirella ». Come unto these Yellow Sands, Four radio Plays by A. Carter.

Newcastle upon Tyne: Bloodaxe Books, 1985.

Carter, Angela. « The Lady of the House of Love ». The Bloody Chamber. Harmondsworth: Penguin Books, 1981.

Davidson, D. « What metaphors mean ». Inquiries into Truth and Interpretation. Oxford: Clarendon Press, 1984.

Derrida, Jacques. « Mythologie blanche, la métaphore dans le texte philosophique ». Marges de la philosophie, Paris : Éditions de Minuit, 1972.

Derrida, Jacques. « Le retrait de la métaphore ». Poésie 7 (1978).

Eco, Umberto. Sémiotique et philosophie du langage. Paris : PUF, « Quadrige », 1988.

Genette, Gérard. «Frontières du récit ». Communications $8:$ L'analyse structurale du récit. Paris : Éditions du Seuil, 1981.

Jakobson, Roman. «Deux aspects du langage et deux types d'aphasie » in Essais de linguistique générale. Paris : Éditions de Minuit, 1963.

Ricoeur, Paul. La Métaphore vive. Paris : Éditions du Seuil, 1975.

Todorov, Tzvetan. Introduction à la littérature fantastique. Paris : Éditions du Seuil, 1970.

\section{NOTES}

1. R. Jakobson, "Deux aspects du langage et deux types d'aphasie", in Essais de linguistique générale, Paris : Éditions de Minuit, 1963, 63.

2. Ibid., 67.

3. Ricœur accuse la linguistique structurale d'un « zèle binariste », d'avoir réduit la complexité et la diversité des tropes pour ne garder que la métaphore et la métonymie (La Métaphore vive, 364).

4. Come unto these Yellow Sands: Four Radio Plays, 7. « Vampirella » fut diffusée par la BBC en 1976.

5. «Vampirella » et « The Lady of the House of Love » sont uniques dans l'oeuvre cartérienne, en ce que la première est la seule pièce à avoir été adaptée en nouvelle. Sa deuxième pièce, "Come Unto these Yellow Sands ", inspirée de la vie du peintre parricide Richard Dadd, est restée sans réécriture; les pièces « Puss in Boots" et «The Company of Wolves" sont des adaptations de contes que Carter avait écrits quelques années auparavant, le second ayant été également adapté cinématographiquement par Neil Jordan. Vers la fin de la vie de Carter, il fut question de faire un film de «Vampirella » avec ce même cinéaste.

6. Devant le spectacle du désarroi de la comtesse, le héros affirme : «Oh I don’t believe your silly tales... just the hysteria of a young girl. In this isolated place, at the back of beyond, with only the family portraits for company ». Dans une référence transparente au «talking cure » de Freud, il remarque : "We should take you away to Vienna, where doctors could examine you. You would stretch out on the therapeutic couch and the stern, quiet, bearded physician would tease from you during the slow intervals of healing time the confused origins of your sickness. » La pièce formule les mêmes suggestions, mais de façon plus humoristique : «yes, perhaps I shall take her 
to Vienna; and we shall clip off her fingernails and take her to a good dentist, to deal with her fangs ».

7. L'ignoble n'est pas loin non plus, dans des expressions telles que « a blot on one's reputation ». 8. Le cannibale Sawney Beane qui livre une diatribe au vitriol contre l'exploitation des pauvres, est présent en sa capacité d'époux de la gouvernante de Vampirella.

9. The Lady of the House of Love, 92 et The Bloody Chamber, 102.

10. La comtesse se pique en effet le doigt sur une écharde. Destiné à panser le flot de sang, ce baiser constitue un geste dépourvu de toute pulsion érotique - «the innocent remedies of the nursery ».

11. Les vêtements que porte Vampirella dans la pièce sont moins connotés - « white muslin dress completed with paisley shawl ». La mousseline et le châle de cachemire sont remplacés par un sous-vêtement diaphane, taché de la marque d'une possible transgression: "a white négligé stained a little with blood".

12. Tzvetan Todorov remarque qu'il est difficile, voire impossible, de cerner l'histoire fantastique par le biais d'un « style » quelconque, et que l'identification des fonctions qu'a le fantastique dans une œuvre se profile comme une démarche plus fructueuse. Voir Introduction à la littérature fantastique, 1970, Paris : Éditions du Seuil, 98.

13. «[L]a désignation la plus sobre des éléments et des circonstances d'un procès peut déjà passer pour une amorce de description ». Gérard Genette, "Frontières du récit ", Communications 8: L'analyse structurale du récit, Paris : Éditions du Seuil, 162.

14. Voir Umberto Eco, Sémiotique et philosophie du langage, Paris : PUF, « Quadrige », 2001, surtout 173-176.

15. Eco remarque d'ailleurs que «parler de la métaphore signifie parler de l'activité rhétorique dans toute sa complexité » (ibid., 139).

16. Paul Ricoeur, La Métaphore vive, 364.

\section{INDEX}

oeuvrecitee Vampirella, Lady of the House of Love (The) 